\title{
Opciones reales implícitas en una empresa distribuidora de alimentos
}

\section{RESUMEN}

El presente estudio, se encuentra enfocado en la valuación de un proyecto mediante opciones reales. Se postula que la flexibilidad que posee este tipo de proyectos, aporta un valor agregado a este, considerando el hecho de poder decidir el momento más apropiado para poder expandirse, dado los distintos escenarios que se pueden dar en el largo plazo. Particularmente, se quiere valorar la opción de diferir en la estrategia de expansión a través de la construcción de sucursales en distintas ciudades del país. Se pudo inferir que la opción real de diferir en un proyecto de expansión generará un valor adicional, principalmente por el beneficio de abrir un establecimiento con anterioridad a 10 programado, considerando el efecto del riesgo y variabilidad de las ventas.

Palabras clave: diferir, opciones, proyectos, reales flexibilidad riesgo

REAL OPTIONS IMPLICIT IN A FOOD DISTRIBUTION COMPANY

\section{ABSTRACT}

The present study focuses on the valuation of a project through real options. It suggests that the flexibility that this type 0 projects have provides a value added to it, considering that the fact of being able to decide the most appropriate time to expand given the different potential scenarios in the long term. In particular, it is desired to valuate the option of deferring the expansion strategy through the construction of branches in various cities of the country It was possible to infer that the real option of postponing an expansion project generates an additional value, mainly due to the benefit of opening an branch before schedule, considering the risk effect and variability.

Keywords: deferring, flexibility, options, project, real, risk

\section{INTRODUCCIÓN}

El presente estudio, se encuentra enfocado en la valuación de un proyecto mediante opciones reales. Se postula que la flexibilidad que posee este tipo de proyectos, da un valor agregado a éste, considerando el hecho de poder decidir el momento más apropiado para poder expandirse dado los distintos escenarios que se pueden dar en el largo plazo, Mascareñas et al (2004). Particularmente, se quiere valorar la opción de diferir en la estrategia de expansión a través de la construcción de sucursales en distintas ciudades del país.

Los proyectos a menudo requieren de un gran presupuesto, que será administrado según los requerimientos de capital, los que influirán directamente en el largo plazo. Este presupuesto va acompañado de las decisiones de inversión del proyecto, como las medidas de expansión, alternativas tecnológicas, reemplazo de los equipos, y las decisiones de compra o arriendo, etc.

El caso de estudio corresponde a una empresa familiar con una trayectoria de más de 30 años en el sur de Chile. Las operaciones de ésta están enfocadas a la distribución de productos de consumo masivo; principalmente alimentos y bebidas, además de artículos de limpieza y de uso en el hogar, todas destinadas a proveer a los comerciantes minoristas de la región y sus alrededores.

Analizando la trayectoria y los resultados de la empresa a lo largo del tiempo, resulta necesario ampliar los horizontes, creando en este caso nuevas sucursales que permitan ampliar el mercado objetivo. Hoy en día, la competencia es muy fuerte, ya que desde hace años, con la llegada masiva de las grandes empresas de supermercados líderes en el rubro, el crecimiento de las ventas ha disminuido notablemente, así como también, han cambiado las necesidades de los clientes, se han diversificado, de manera tal que es necesario reinventar el sistema.

\section{MARCO TEÓRICO}

\subsection{Visión tradicional de evaluación de proyectos}

Desde hace tiempo, los evaluadores de proyectos han sabido que muchos de sus pronósticos "siempre" están errados. Las fuerzas

\footnotetext{
Doctor en Finanzas, Master en finanzas Académico del Departamento de Economía y Finanzas de la Universidad del Bío Bío Chile. E-mail: mauroguti40@yahoo.es

** Máster en Gestión Industrial Universidad de Concepción Chile. E-mail: gonzalezs.jorge@gmail.com
} 
del mercado y la volatilidad generan cargas para las estimaciones, con frecuencia, difieren mucho de las que se anticipan en los planes originales. La evaluación de las inversiones a largo plazo presenta un desafío mayor, ya que se caracterizan por largos periodos de recuperación, incertidumbre y condiciones del negocio que cambian en el transcurso del proyecto. Ante esto, solo unas pocas estimaciones son utilizadas para determinar las decisiones de presupuesto de capital, por ejemplo el método del valor actual neto (VAN), la tasa interna de retorno (TIR), periodo de recuperación de inversión (PRI). EI VAN y la TIR, combinados con los flujos de fondos descontados (DCF por su siglas en inglés) son ampliamente aceptados como herramientas para la toma de decisiones. DCF estima los ingresos y egresos de una inversión descontada a una tasa acorde al riesgo del proyecto. Esta estimación asume que la inversión no puede posponerse y una vez iniciada no puede alterarse en el transcurso del proyecto, lo cual en la realidad no siempre es así, ya que los proyectos generalmente poseen la opción y no la obligación de seguir adelante con las inversiones, por lo tanto, esta estimación no valora esta flexibilidad, que genera un valor agregado al proyecto.

\subsection{Teorías}

El análisis de las opciones reales presenta una alternativa atractiva en la valuación de proyectos, debido a que explícitamente valora la flexibilidad futura de la opción de invertir dado un cierto escenario (Amram \& Kulatilaka, 1999; Smith \& McCardle, 1998; Trigeorgis, 1996). El objetivo de este estudio es analizar la flexibilidad en las estrategias de expansión con opciones reales.

Las opciones reales, son derechos que las empresas poseen sobre determinados activos y que les permiten adaptarse al entorno con mayor flexibilidad, soportando menores riesgos. Desde la perspectiva de las opciones reales, el activo total de la empresa se compone de las inversiones en funcionamiento y de sus oportunidades futuras de inversión. Dicho de otro modo, el valor global de un proyecto de inversión en la actualidad, llamémoslo VAN total (mientras que denominaremos VAN básico al clásico valor actual neto), será igual a:

$$
\begin{aligned}
\text { VAN total }= & \text { VAN básico } \\
\text { implícitas }) & \text { VA }
\end{aligned}
$$

En términos generales las variables a considerar para la valoración de la opción real, son:
- Precio del activo, valor actual de los flujos de caja esperados del proyecto

- Precio del ejercicio, costo de la inversión para ejercer la opción del proyecto.

- Riesgo del activo, volatilidad esperada del rendimiento del proyecto

- El tipo de interés libre de riesgo

- Tiempo hasta el vencimiento.

Las opciones reales que puedan encontrarse en la realidad de las empresas se pueden agrupar en grupos.

- Diferir/Aprender

- Inversión/Crecimiento

- Intercambio

- Desinvertir/Reducir.

Por último, dentro de los métodos básicos para valorizar opciones tenemos:

- Modelo de Black Scholes

- Modelo binomial

- Simulación de Montecarlo

\section{APLICACIÓN DE LA METODOLOGÍA}

\subsection{Antecedentes generales del proyecto}

La aplicación práctica de la metodología mencionada, se basa en la estrategia de expansión de una distribuidora de alimentos localizada en el sur de Chile, la cual actualmente solo posee una sucursal y trabaja a nivel provincial. El proyecto contempla principalmente la estrategia de expansión mediante una nueva sucursal en otra ciudad de Chile. A continuación se presenta información básica:

Tabla 1. Pronósticos de mercados según provincia del sur de Chile

\begin{tabular}{|c|c|c|c|c|c|}
\hline \multicolumn{5}{|c|}{ Tamaño Mercado en comparado con Ñuble } \\
\hline Provincia & Ñuble & Linares & $\begin{array}{c}\text { Concep- } \\
\text { ción }\end{array}$ & Bío Bío & Arauco \\
\hline Tamaño \% & $100 \%$ & $94 \%$ & $158 \%$ & $89 \%$ & $40 \%$ \\
\hline
\end{tabular}

Entre las conclusiones, se menciona que la incursión en este tipo de proyectos genera un cambio estructural y organizacional que dan pie a inicio de nuevas sucursales y así construir una 
cadena de sucursales. De esta manera la opción real se presenta en el poder esperar y ver la evolución de las ventas, para instalar otra sucursal, ya que, se tiene la opción y no el deber de construir otra. Por la dinámica del proyecto la opción cae en la categoría de "Diferir y Aprendizaje".

La opción de diferir un proyecto de inversión proporciona a su propietario el derecho a posponer su realización durante un plazo de tiempo determinado. Esta opción genera valor, solo si la empresa tiene derechos exclusivos para invertir en un proyecto. Además esta pierde valor conforme pasa el tiempo y las barreras de entrada al mercado desaparezcan. El derecho exclusivo está asociado al sello y prestigio de la empresa, el cual no puede ser imitado, no es de libre disociación, es decir, el nombre y lo que éste conlleva no puede ser utilizado por un tercero, con el fin de ganar prestigio a costa de una marca o sello consolidado. Como supuesto relevante se considera que al menos existe un terreno disponible para invertir en alguna de las provincias estudiadas. A continuación se determinaran las principales variables del caso estudiado.

\subsection{Estimación de Ventas}

Se considera utilizar el "índice de ventas de supermercado" (ISUP) (INE, 201211), debido a dos razones, primero como se observa en el grafico 1 y 2 existe una fuerte correlación de Pearson ${ }^{2}$ igual a $79,7 \%$. Segundo, el ISUP posee una historia de 21 años y contempla un promedio nacional, entregando más información. Además con este indicador, se podría generar mejores estimaciones de escenarios futuros, comparado con las ventas locales, si se considera que este indicador incorpora el efecto variables que afectan a las ventas en otras ciudades.

Al considerar una relación entre estas dos variables, se podría decir que al estimar valores futuros para el ISUP también estaríamos estimando ventas futuras para las nuevas sucursales, Sapag (2007). Para ello se modela el riesgo de la demanda mediante una simulación de Montecarlo, con esto es necesario decidir que distribución de probabilidad se debe utilizar para estimar la demanda. Cabe recordar que el objetivo es generar números aleatorios con una distribución, para incorporarlo en el modelo y generar y entender las variables aleatorias a través de tiempo (procesos estocásticos).

Gráfico 1. Ventas totales reales junto con ISUP

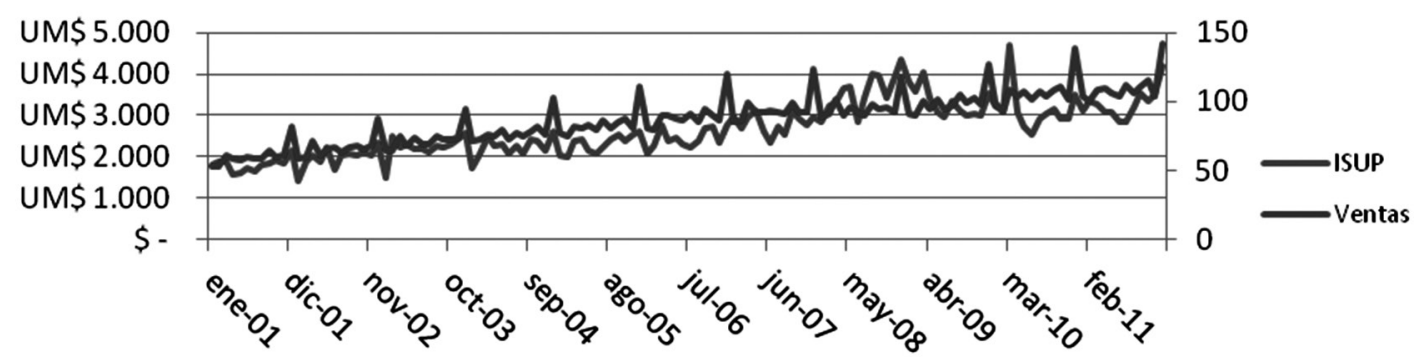

Gráfico 2. Relación lineal con datos anuales. Ecuación lineal $1 Y=30,448 X R^{2}=0,80$

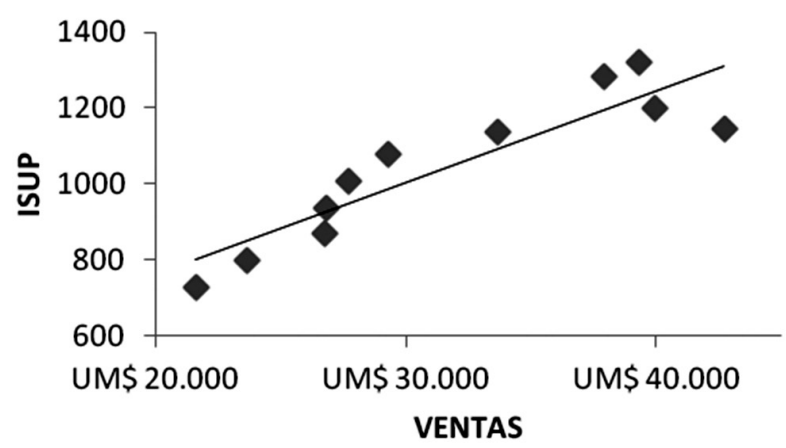

Instituto Nacional de Estadística de Chile

2 En estadística, el coeficiente de correlación de Pearson es un índice que mide la relación lineal entre dos variables aleatorias cuantitativa. 
El modelo estocástico a utilizar será Geometric Brownian Motion, también conocido como Movimiento Browniano, es utilizado para estimar los retornos de S\&P500, el movimiento del polen en el agua o el caminar de un hombre en condiciones de ebriedad. La tasa de cambio de un promedio es browniana, no así las observaciones subyacentes, por ejemplo el precio de las acciones no necesariamente siguen un movimiento browniano, pero si su retornos. Este es utilizado para estimar el comportamiento del precio de las acciones en teoría financiera y en otras incertidumbres, Cardin (2007).

Matemáticamente el movimiento browniano se define de la siguiente manera:

$$
d S=\mu S d t+\tilde{o} S d z
$$

En donde $S$ es el valor del activo, $\mu$ es la tendencia de retorno anual, õ es la volatilidad del precio del activo, y $d z$ es la "choque aleatorio" aplicado a $\mu$.
Para nuestro caso en particular el precio del activo, serian las ventas, y la tasa de retorno anual, seria la tasa de crecimiento esperado de las ventas.

Conocer el modelo teórico es sólo el comienzo, ya que hay que calibrarlo apropiadamente $(\mu, \tilde{o})$, por lo cual se realiza una regresión lineal a la serie, suavizada mediante logaritmo natural, considerando un período anual, obteniendo el gráfico 3 , en donde se observa que la tasa de crecimiento anual promedio es de $7,44 \%$ y una desviación estándar de $5,88 \%$.

Considerando los valores de $(\mu$, õ), igual a $(7,44 \%$ $5,88 \%)$ y un ISUP base de $1.322,45$ puntos correspondiente al último año, se pueden generar pronósticos mediante la ecuación 2 , generando números aleatorios mediante el inverso de la distribución acumulativa normal para la media y desviación estándar especificadas, los resultados para un pronóstico de 5 años se encuentran en la tabla 2.

Grafico 3. Relación lineal con datos anuales. Ecuación lineal 3: $y=0,0744 x+5,815 R^{2}=0,984$

Estimación de Parámetros

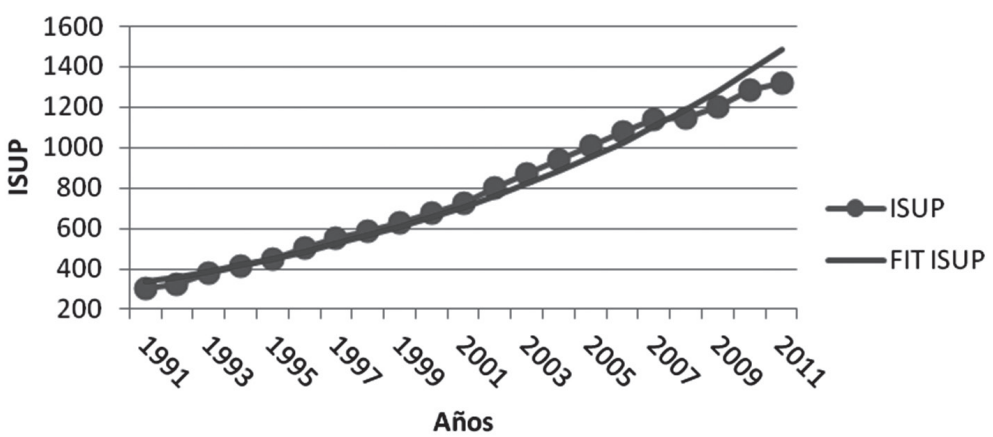

Tabla 2. Crecimientos esperados. ISUP $2011^{*}$ Último valor registrado

$$
\begin{aligned}
& d I S U P=\mu I S U P_{2011} d t+\sigma I S U P_{2011} d z=I S U P_{2011}(\mu d t+\sigma d z) \\
& d I S U P=1322,45 *(7,44 \%+5,88 \%)=12,95 \\
& {I S U P_{2012}}=I S U P_{2011}+d I S U P=1322,45+12,95=1335,40
\end{aligned}
$$

\begin{tabular}{|c|c|c|c|}
\hline Años & $\begin{array}{c}\text { ISUP } \\
\text { Pronóstico }\end{array}$ & $\begin{array}{c}\text { Número aleatorio de una } \\
\text { distribución normal estandarizada }\end{array}$ & $\begin{array}{c}\text { Tasa de crecimiento (Tasa de } \\
\text { crecimiento anual promedio + } \\
\text { Número aleatorio * Volatilidad) }\end{array}$ \\
\hline $2011^{*}$ & $1.322,45$ & $-1,09839621$ & 0,00978922 \\
\hline 2012 & $1.335,40$ & $-0,61713891$ & 0,03809814 \\
\hline 2013 & $1.386,27$ & 0,73740143 & 0,11777604 \\
\hline 2014 & $1.549,54$ & 0,34148105 & 0,09448688 \\
\hline 2015 & $1.695,95$ & 0,14389622 & 0,08286438 \\
\hline
\end{tabular}


Por último, considerando la información de estudios previos del proyecto, se recuerda la tasa de aprendizaje que castiga las ventas en un $35 \%$ y $17,5 \%$ para el primer y segundo año respectivamente, al momento de abrir la nueva sucursal, además de los pronósticos de mercados según provincia. Por ende, para estimar las ventas de un local, primero se pronostica según la tasa de crecimiento del ISUP, luego se procede a aplicar la tasa de castigo, y luego se le aplica la tasa mercado según la provincia.

Así de esta forma, se consideran ajustes de aprendizaje, valores macroeconómicos (ISUP), y los efectos de participar en distintos mercados, como esfuerzos para estimar las futuras ventas en las nuevas sedes.

Consecuentemente a éstas estimaciones, se realiza una validación a los pronósticos con el propósito de verificar la exactitud de éstos, con los valores observados, por lo que se consideran valores en base a la información hasta el 2005 y se estiman 6 valores correspondientes a los años 2006, 2007, 2008, 2009, 2010 y 2011 tal como muestra el gráfico 4 , sin embargo esto es sólo una simulación de los valores futuros del ISUP y al repetir el ejercicio se arrojan otros escenarios, por lo tanto se realiza un promedio de 3500 simulaciones para examinar la exactitud del modelo, arrojando los siguientes resultados (Yuan (2009):
Tabla 3. Resultados Test de pronósticos

\begin{tabular}{|c|c|}
\hline Parámetro & Valor \\
\hline Desviación Estándar & $14,76 \%$ \\
\hline Máx. Desv. Estándar & $33,40 \%$ \\
\hline Mín Desv. Estándar & $1,91 \%$ \\
\hline
\end{tabular}

Como principal conclusión de la validación es que no es conveniente simular más de 6 años, dado que el error se dispara a medida que vamos avanzando en el tiempo. Por lo tanto para efectos de este estudio, se harán pronósticos mediante una simulación para 5 años y luego se asumirá un ISUP constante en el tiempo, con el fin de no sobrevaluar el proyecto, así como se muestra en el grafico 5.

\subsection{Estimación de tasa de descuento}

Por último considerando la ecuación 3 para estimar el costo de capital promedio ponderado, se puede estimar una tasa de descuento, con la información disponible, aplicando un impuesto del $20 \%$ de primera categoría para este proyecto, Copeland y Weston (2003).

$$
W A C C=k_{s} * \frac{S}{B+S}+k_{d} *\left(1-\tau_{c}\right) * \frac{B}{S+B}
$$

Gráfico 4. Simulación test de pronósticos 2006-2011.

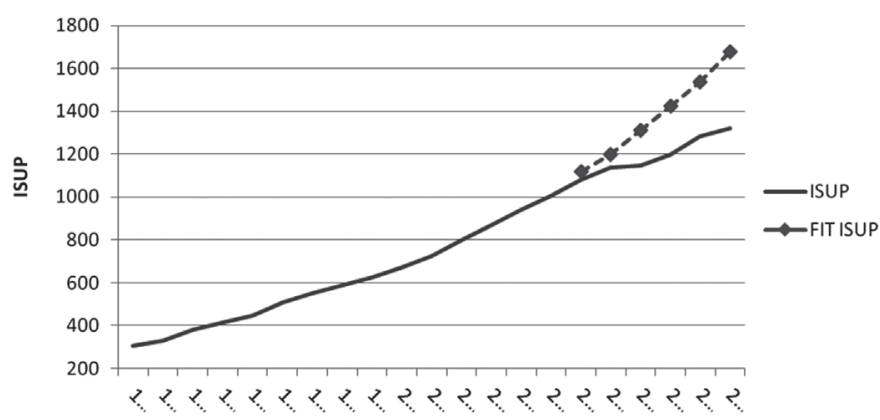

Gráfico 5. Simulación de pronósticos 2012-2022.

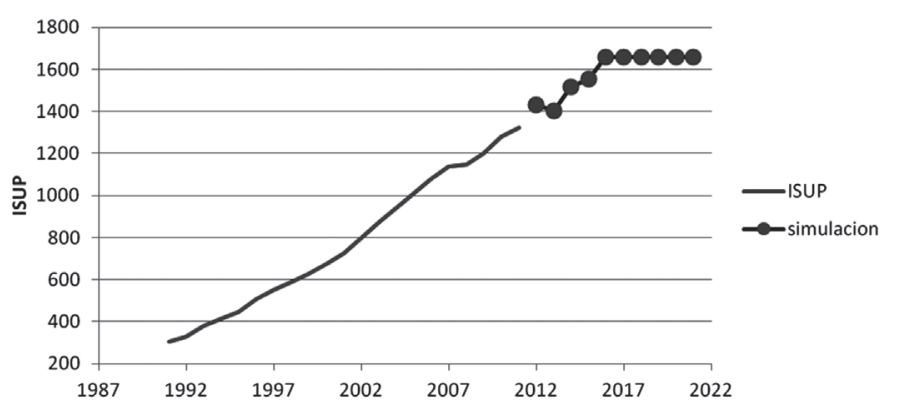


Tabla 4. Resumen caculo WACC

\begin{tabular}{|c|c|}
\hline Ítem & Valor \\
\hline Deuda/capital (A. Damodaran) & $41,34 \%$ \\
\hline Tasa de interés $(T c)$ & $20,00 \%$ \\
\hline Costo deuda $(K d)$ & $4,29 \%$ \\
\hline \% Deuda & $29,25 \%$ \\
\hline \% Capital & $70,75 \%$ \\
\hline Costo de capital $(K s)$ & $10,85 \%$ \\
\hline WACC (real) & $8,68 \%$ \\
\hline
\end{tabular}

Por lo tanto, bajo los supuestos y consideraciones señalas en este punto, se puede considerar una tasa real anual del $8,68 \%$ para el proyecto planteado en el estudio.

\subsection{Simulación y resultados}

Como se puede apreciar en el punto 3.2, se generan estimaciones de ventas para cada ciudad en base a las estimaciones del rendimiento anual del ISUP, tal como se mostró en la tabla 1, aplicando además los ajustes señalados de aprendizaje y la participación en los distintos mercados (Provincias). En este punto, se destaca que la apertura de nuevas sucursales será mediante la reinversión, en otras palabras, las nuevas sedes se financiarán mediante los ingresos percibidos por las nuevas sucursales. Prefiriendo la inversión en los mercados más grandes, por lo que el orden de las inversiones será, Concepción, Linares, y Bío Bío, al sur de Chile.

En primer lugar se deben generar distintos números aleatorios para las distintas provincias

Luego se aplican los números aleatorios a la ecuación 2 y se generan los resultados del la tabla 6 , como las tasas de crecimientos esperadas para el ISUP en casa región, cabe destacar que se realizan estimaciones de venta por cada sucursal.

Luego se realizan las estimaciones de las ventas, según tabla 7 , respetando las correcciones mencionadas en el punto 3.2 , se calculan las siguientes demandas.

Con las ventas estimadas y los costos asociados discutidos en el punto 3.2, se procede a armar los flujos contables y de caja para cada ciudad (Copeland t Weston (2003), tomando el escenario con y sin opción real en el proyecto. Para el escenario sin opción real, se considera la apertura de los locales bajo una demanda en que se consideran los promedios de crecimiento para cada ciudad sin el componente de aleatoriedad y sólo se aplica una tasa de crecimiento constante calculada anteriormente del $7,44 \%$ por lo tanto se genera el escenario de demanda mostrado a continuación.

Se generan 2000 escenarios para los valores que puede tomar el VAN del proyecto (Tabla 10). Se considera un VAN o NPV por sus siglas en inglés, en primer lugar de un proyecto con opción real $\left(N P V_{1}\right)$ y en segundo lugar un proyecto sin opción real $\left(\mathrm{NPV}_{2}\right)$

Tabla 5. Generación de números aleatorios

\begin{tabular}{|c|c|c|c|c|}
\hline Años & $\begin{array}{c}\mathbf{N}^{\circ} \text { Aleatorio } \\
\text { NUUBLE }\end{array}$ & $\begin{array}{c}\mathbf{N}^{\circ} \text { Aleatorio } \\
\text { LINARES }\end{array}$ & $\begin{array}{c}\mathbf{N}^{\circ} \text { Aleatorio } \\
\text { CONCEPCIÓN }\end{array}$ & $\begin{array}{c}\mathbf{N}^{\circ} \text { Aleatorio } \\
\text { Biobío }\end{array}$ \\
\hline 2012 & 0,030 & $-0,257$ & 0,465 & 1,082 \\
2013 & 0,042 & $-0,117$ & 0,470 & $-2,714$ \\
2014 & $-0,243$ & 0,725 & $-0,873$ & 1,822 \\
2015 & 0,686 & $-2,100$ & $-0,854$ & 0,871 \\
2016 & 1,280 & 0,122 & $-1,252$ & $-0,142$ \\
\hline
\end{tabular}

Tabla 6. Tasa de crecimiento ISUP por ciudad

\begin{tabular}{|c|c|c|c|c|}
\hline Años & Nuble & Linares & Concepción & Bío Bío \\
\hline 2012 & $7,61 \%$ & $5,93 \%$ & $10,18 \%$ & $13,80 \%$ \\
\hline 2013 & $7,69 \%$ & $6,75 \%$ & $10,20 \%$ & $-8,53 \%$ \\
\hline 2014 & $6,01 \%$ & $11,70 \%$ & $2,30 \%$ & $18,16 \%$ \\
\hline 2015 & $11,47 \%$ & $-4,92 \%$ & $2,42 \%$ & $12,56 \%$ \\
\hline 2016 & $14,97 \%$ & $8,16 \%$ & $0,07 \%$ & $6,60 \%$ \\
\hline
\end{tabular}


Tabla 7. Escenarios de ventas por ciudad

\begin{tabular}{|c|c|c|c|c|c|c|}
\hline & AÑO & ISUP & Ñuble & Linares & Concepción & Bío Bío \\
\hline \multirow{5}{*}{ 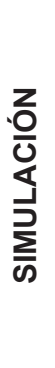 } & 2012 & 1423 & 42.271 & 40.093 & 45.562 & 40.783 \\
\hline & 2013 & 1533 & 45.490 & 42.800 & 63.729 & 37.306 \\
\hline & 2014 & 1625 & 48.986 & 31.077 & 85.129 & 44.079 \\
\hline & 2015 & 1811 & 51.929 & 37.505 & 87.088 & 49.617 \\
\hline & 2016 & 2082 & 57.887 & 49.170 & 89.192 & 34.381 \\
\hline \multirow{3}{*}{ 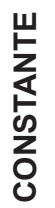 } & 2017 & 2082 & 57.887 & 49.170 & 89.192 & 34.381 \\
\hline & .. & $\cdots$ & $\cdots$ & $\cdots$ & $\cdots$ & $\cdots$ \\
\hline & 2021 & 2082 & 57.887 & 49.170 & 89.192 & 34.381 \\
\hline
\end{tabular}

Tabla 8. Tasa de crecimiento constante ISUP por ciudad

\begin{tabular}{|c|c|c|c|c|}
\hline Time & Nuble & Linares & Concepción & Bío Bío \\
\hline 2012 & 0,0744 & 0,0744 & 0,0744 & 0,0744 \\
\hline 2013 & 0,0744 & 0,0744 & 0,0744 & 0,0744 \\
\hline 2014 & 0,0744 & 0,0744 & 0,0744 & 0,0744 \\
\hline 2015 & 0,0744 & 0,0744 & 0,0744 & 0,0744 \\
\hline 2016 & 0,0744 & 0,0744 & 0,0744 & 0,0744 \\
\hline
\end{tabular}

Tabla 9. Escenarios de ventas por ciudad a tasa constante

\begin{tabular}{|c|c|c|c|c|c|c|}
\hline & AÑO & ISUP & Ñuble & Linares & Concepción & Bío Bío \\
\hline \multirow{5}{*}{ 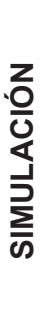 } & 2012 & 1421 & 1.421 & 40.666 & 44.430 & 38.503 \\
\hline & 2013 & 1527 & 1.527 & 43.692 & 60.587 & 41.368 \\
\hline & 2014 & 1640 & 1.640 & 30.512 & 78.903 & 44.445 \\
\hline & 2015 & 1762 & 1.762 & 41.609 & 84.773 & 47.752 \\
\hline & 2016 & 1893 & 1.893 & 54.187 & 91.080 & 33.348 \\
\hline \multirow{5}{*}{ 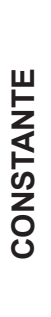 } & 2017 & 1893 & 1.893 & 54.187 & 91.080 & 33.348 \\
\hline & 2018 & 1893 & 1.893 & 54.187 & 91.080 & 33.348 \\
\hline & 2019 & 1893 & 1.893 & 54.187 & 91.080 & 33.348 \\
\hline & 2020 & 1893 & 1.893 & 54.187 & 91.080 & 33.348 \\
\hline & 2021 & 1893 & 1.893 & 54.187 & 91.080 & 33.348 \\
\hline
\end{tabular}


Tabla 10. Distintos escenarios del proyecto

\begin{tabular}{|c|c|c|}
\hline Escenario & $\mathrm{NPV}_{1}$ & $\mathrm{NPV}_{2}$ \\
\hline 1 & UM \$ 263.707 & UM \$ 164.912 \\
\hline 2 & UM \$ 235.666 & UM \$ 177.152 \\
\hline 3 & UM \$ 206.076 & UM \$ 208.572 \\
\hline 4 & UM \$ 310.933 & UM \$ 148.824 \\
\hline 5 & UM \$ 324.709 & UM \$ 187.788 \\
\hline 6 & UM \$ 202.407 & UM \$ 229.273 \\
\hline 7 & UM \$ 284.450 & UM \$ 174.138 \\
\hline 8 & UM \$ 180.610 & UM \$ 164.523 \\
\hline$\ldots$ & UM \$ 284.894 & UM \$ 225.083 \\
\hline & UM \$ 207.325 & UM \$ 222.132 \\
\hline 1999 & UM \$ 191.002 & UM \$ 171.541 \\
\hline 2000 & UM \$ 284.884 & UM \$ 191.696 \\
\hline
\end{tabular}

De estos distintos escenarios se pueden obtener estadísticas: valor máximo, valor mínimo, valor promedio, histograma, función de distribución acumulada para ambos proyectos presentados a continuación.

Tabla 11. Distintos escenarios del proyecto

\begin{tabular}{|c|c|c|}
\hline Proyecto & Estadístico & Valor \\
\hline \multirow{3}{*}{ NPV1 } & Promedio & UM\$ 218.379 \\
\cline { 2 - 3 } & Máximo & UM\$ 381.684 \\
\cline { 2 - 3 } & Mínimo & UM\$ 122.685 \\
\hline \multirow{3}{*}{ NPV2 } & Promedio & UM\$ 191.452 \\
\cline { 2 - 3 } & Máximo & UM\$ 271.816 \\
\cline { 2 - 3 } & Mínimo & UM\$ 126.870 \\
\hline
\end{tabular}

Gráfico 6. Histograma del proyecto con opción real.

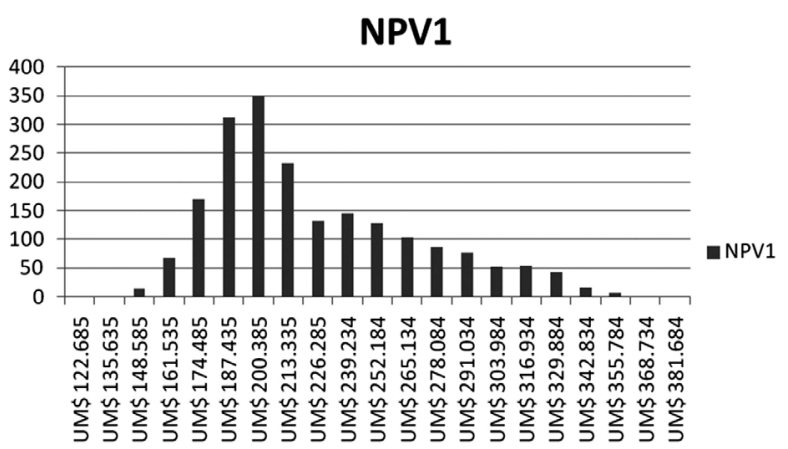

El análisis, se basa en la información mostrada en gráficos y tablas en los puntos anteriores que comparan los escenarios de valuación con y sin opción real.

Como se pudo observar, tanto la media como el máximo del proyecto con opción real es mayor, además mediante el histograma vemos que la frecuencia de los NPV se desplaza hacia derecha, implicando un mayor valor para el proyecto. Esto se aprecia adecuadamente si se grafíca la función de distribución acumulada (grafico 6) en donde se ve claramente que el valor del proyecto se incrementa ante la opción de crecimiento.

Esto refleja un efecto positivo del proyecto al adelantar la instalación de una sucursal ante escenarios de positivos o el efecto de esperar y diferir el momento en que se abre una sucursal por ventas bajo los promedios, lo cual repercute en la valuación del proyecto de forma significativa en escenarios positivos pero no así en escenarios menos favorables de ventas, de esta manera el beneficio que entrega la opción real, eleva la valuación en promedio en un $40,4 \%$.

\section{CONCLUSIONES}

En primer lugar, se mostró una metodología que mediante una planilla de cálculo permite valorar una opción real, además proveer información sobre la manera en la cual un diseño de estimación de ingresos puede flexibilizarse, sin tener que tomar supuestos financieros.

Se considera que la opción real solo puede ser ejercida una vez al año, y tarda un año una vez realizadas las inversiones para poder recibir los beneficios del proyecto. Esto se define así ya que a pesar de que la decisión de expansión puede ser tomada en cualquier momento, el desarrollo

Gráfico 7. Histograma del proyecto sin opción real.

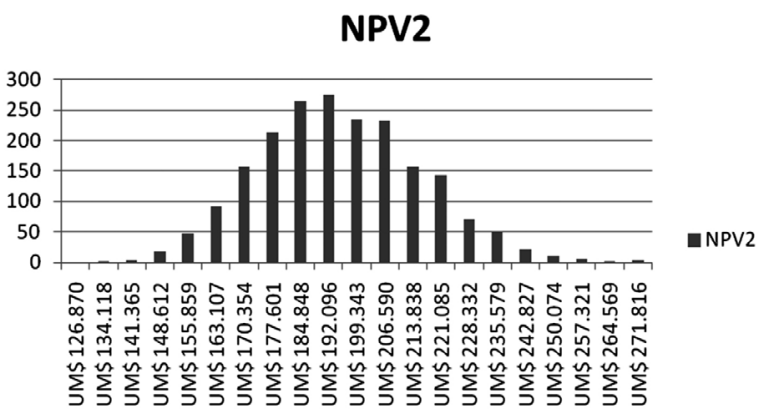


Gráfico 8. Gráfico de Función de distribución acumulada.

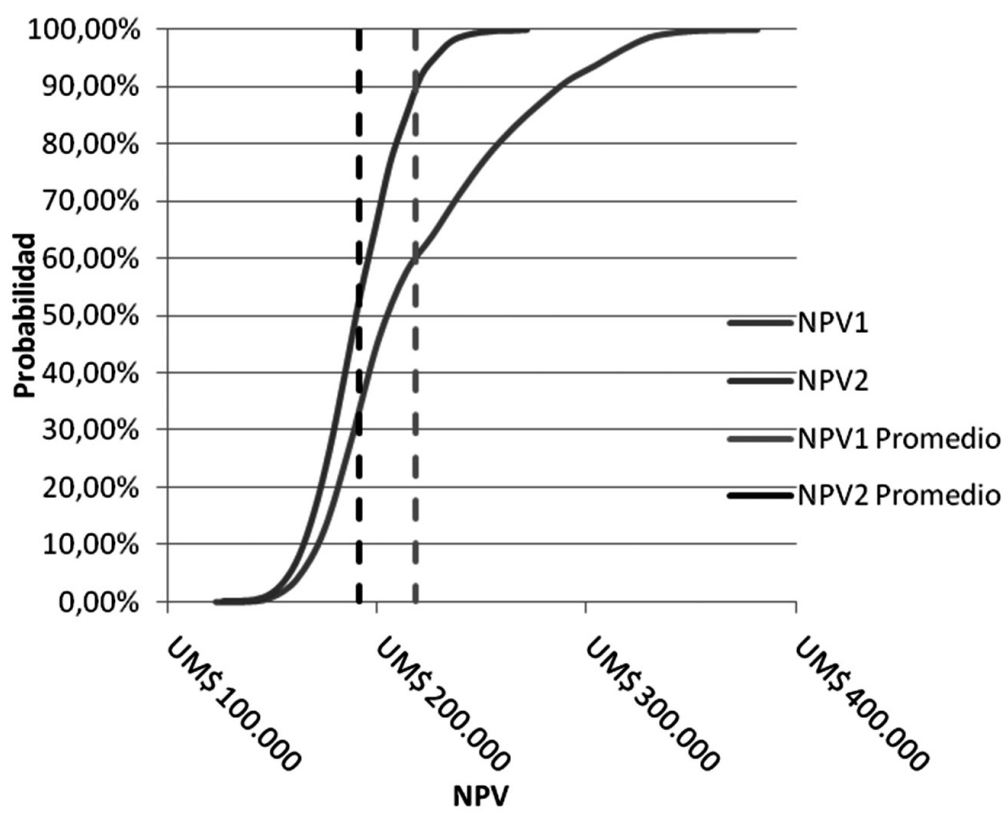

del proyecto tarda un tiempo estimable a un año, presentando características de una opción europea.

Se pudo inferir que la opción real de diferir en un proyecto de expansión genera un valor adicional, principalmente por el beneficio de abrir un establecimiento con anterioridad a lo programado, considerando el efecto del riesgo y variabilidad de las ventas que pueden darse en distintos escenarios, es decir, que si en el transcurso del proyecto éste es aceptado por la demanda (escenario positivo) la empresa se expande de forma más agresiva y adelanta las inversiones de nuevos locales, y de esta manera percibe más ingresos, en comparación a un escenario en que no se tenga esta opción de adelantar las inversiones. En otras palabras minimiza la exposición al riesgo al invertir de forma diferida, maximizando el potencial de ganancia en circunstancias favorables.

\section{REFERENCIAS}

[1] Amram, M., \& Kulatilaka, N. (1999). Real Options: Managing Strategic Investment in an Uncertain World, Harvard Business School Press, Boston, MA, 1999.

[2] Cardin, Michel-Alexandre. (2007) "FACING REALITY: DESIGN AND MANAGEMENT OF FLEXIBLE ENGINEERING SYSTEMS". Massachusetts Institute of Technology.
[3] Copeland, Thomas E., J. Fred Weston, and Kuldeep Shastri. Financial Theory and Corporate Policy. 4th ed. Prentice Hall, 2003.

[4] Damodaran, Aswath. "Discount Rate Estimation", 2012. http://pages.stern.nyu. edu/ adamodar/.

[5] Instituto Nacional de Estadísticas, ed. "METODOLOGÍA ÍNDICE DE VENTAS DE SUPERMERCADO". Sub departamento de Estadísticas Coyunturales de Comercio y Servicios, Chile 2012.

[6] Mascareñas J Lamothe P Luna M Opciones reales y valoración de activos : cómo medir la flexibilidad operativa en la empresa. Madrid: Pearson Educación, 2004

[7] Sapag, Nassir. Preparación y evaluación de proyectos. McGraw-Hill Interamericana Editores S.A. de C.V., 2007.

[8] Smith, J. E., \& McCardle, K. F. (1998). Valuing oil properties: integrating option pricing and decision analysis approaches. Operations Research, 46(2), 198-217.

[9] Trigeorgis, L. (1996). Evaluating leases with complex operating options. European Journal of Operational Research, 91(2), 315-329.

[10]Yuan, Fong-Ching.(2009) Simulationoptimization mechanism for expansion strategy using real option theory». Expert Syst. Appl. 36, no. 829-837. 